\title{
PERIODIC ORBITS OF A NON-AUTONOMOUS QUADRATIC DIFFERENTIAL SYSTEM OBTAINED FROM THIRD-ORDER DIFFERENTIAL EQUATIONS
}

\author{
JAUME LLIBRE $^{1}$ AND AMAR MAKHLOUF ${ }^{2}$
}

\begin{abstract}
We provide sufficient conditions for the existence of periodic solutions of the third-order differential equations $u^{\prime \prime \prime}+\left(a_{1} u+a_{0}\right) u^{\prime \prime}+\left(b_{1} u+b_{0}\right) u^{\prime}+c_{2} u^{2}+a_{0} b_{0} u=\varepsilon^{2} F\left(t, u, u^{\prime}, u^{\prime \prime}, \varepsilon\right)$, where $a_{0}, a_{1}, b_{0}, b_{1}, c_{2}$ are real arbitrary parameters with $b_{0}>0, \varepsilon$ is a small parameter and $F$ is a $\frac{2 \pi}{\sqrt{b_{0}}}$-periodic nonlinear function in the variable $t$. The prime denotes derivative with respect to the independent variable $t$. Moreover we provide an application.
\end{abstract}

\section{INTRODUCTION AND STATEMENT OF THE MAIN RESULTS}

Quadratic differential systems in $\mathbb{R}^{3}$ are some of the simplest systems after linear systems and have been extensively studied in the last years. There are many examples of such systems, see for instance Lorenz system [12], the Chen system [4], the Liu system [10], the Rössler system [16], the Rikitake system [14], the Lu system [9], among several others.

One of the most interesting problems related with quadratic differential systems is the study of their limit cycles, i.e. of their isolated periodic orbits of the system. It is known that every quadratic differential system in $\mathbb{R}^{2}$ has finitely many limit cycles, see for instance [1], [6], [8]. For quadratic systems in $\mathbb{R}^{n}$ with $n>2$ the scenario is very different. There are quadratic systems for $n>2$ with infinitely many limit cycles, see for instance [7].

In [5] the authors study the nonlinear dynamics including the periodic orbits of a quadratic differential system in $\mathbb{R}^{3}$ which comes from from a third-order differential equation. More precisely, they analyze the third-order differential equations

$$
u^{\prime \prime \prime}+\left(a_{1} u+a_{0}\right) u^{\prime \prime}+\left(b_{1} u+b_{0}\right) u^{\prime}+c_{2} u^{2}+c_{1} u+c_{0}=0 .
$$

The goal of this paper is to study the periodic solutions of the third-order nonautonomous differential equations

$$
u^{\prime \prime \prime}+\left(a_{1} u+a_{0}\right) u^{\prime \prime}+\left(b_{1} u+b_{0}\right) u^{\prime}+c_{2} u^{2}+a_{0} b_{0} u=\varepsilon^{2} F\left(t, u, u^{\prime}, u^{\prime \prime}, \varepsilon\right),
$$

where $a_{0}, a_{1}, b_{0}, b_{1}, c_{2}$ are real arbitrary parameters with $b_{0}>0, \varepsilon$ is a small parameter and $F$ is a $\frac{2 \pi}{\sqrt{b_{0}}}$-periodic nonlinear function in the variable $t$. The prime denotes derivative with respect to the independent variable $t$.

2000 Mathematics Subject Classification. 37G15, 37C80, 37C30.

Key words and phrases. periodic orbit, third-order differential equation, quadratic system, averaging theory. 
Our main result on the periodic solutions of the third-order non-autonomous differential equation (1) is the following one.

Theorem 1. Assume that $b_{0}>0$ in the differential equation (1). We define

$$
\begin{aligned}
& \mathcal{F}_{1}\left(X_{0}, Y_{0}\right)=\frac{\sqrt{b_{0}}}{2 \pi} \int_{0}^{\frac{2 \pi}{\sqrt{b_{0}}}} \cos \left(\sqrt{b_{0}} t\right)\left(F_{0}(t, A(t), B(t), C(t))+D(t)\right) d t, \text { and } \\
& \mathcal{F}_{2}\left(X_{0}, Y_{0}\right)=-\frac{\sqrt{b_{0}}}{2 \pi} \int_{0}^{\frac{2 \pi}{\sqrt{b_{0}}}} \sin \left(\sqrt{b_{0}} t\right)\left(F_{0}(t, A(t), B(t), C(t))+D(t)\right) d t,
\end{aligned}
$$

where $F_{0}(t, x, y, z)$ is the term or order zero in $\varepsilon$ when we expand in Taylor series the function $F(t, \varepsilon x, \varepsilon y, \varepsilon z, \varepsilon)$ at $\varepsilon=0$, and

$$
\begin{aligned}
A(t)= & \frac{\left(-\sqrt{b_{0}} X_{0}+a_{0} Y_{0}\right) \cos \left(\sqrt{b_{0}} t\right)+\left(a_{0} X_{0}+\sqrt{b_{0}} Y_{0}\right) \sin \left(\sqrt{b_{0}} t\right)}{\sqrt{b_{0}}\left(a_{0}^{2}+b_{0}\right)}, \\
B(t)= & \frac{\left(\sqrt{b_{0}} Y_{0}+a_{0} X_{0}\right) \cos \left(\sqrt{b_{0}} t\right)+\left(\sqrt{b_{0}} X_{0}-a_{0} Y_{0}\right) \sin \left(\sqrt{b_{0}} t\right)}{a_{0}^{2}+b_{0}}, \\
C(t)= & \frac{\left(b_{0} X_{0}-a_{0} \sqrt{b_{0}} Y_{0}\right) \cos \left(\sqrt{b_{0}} t\right)-\sqrt{b_{0}}\left(a_{0} X_{0}+\sqrt{b_{0}} Y_{0}\right) \sin \left(\sqrt{b_{0}} t\right)}{a_{0}^{2}+b_{0}}, \\
D(t)= & \frac{1}{b_{0}\left(a_{0}^{2}+b_{0}\right)^{2}}\left[\left(\sqrt{b_{0}} X_{0}-a_{0} Y_{0}\right) \cos \left(\sqrt{b_{0}} t\right)-\left(a_{0} X_{0}+\sqrt{b_{0}} Y_{0}\right) \sin \left(\sqrt{b_{0}} t\right)\right] \\
& {\left[\left(\sqrt{b_{0}}\left(a_{1} b_{0}+a_{0} b_{1}-c_{2}\right) X_{0}+\left(b_{0} b_{1}+a_{0}\left(-a_{1} b_{0}+c_{2}\right)\right) Y_{0}\right) \cos \left(\sqrt{b_{0}} t\right)+\right.} \\
& \left.\left(\left(b_{0} b_{1}+a_{0}\left(-a_{1} b_{0}+c_{2}\right)\right) X_{0}+\sqrt{b_{0}}\left(-a_{1} b_{0}-a_{0} b_{1}+c_{2}\right) Y_{0}\right) \sin \left(\sqrt{b_{0}} t\right)\right] .
\end{aligned}
$$

If the function $F$ is $\frac{2 \pi}{\sqrt{b_{0}}}$-periodic in the variable $t$, then for every $\left(X_{0}{ }^{*}, Y_{0}{ }^{*}\right)$ solution of the system

$$
\mathcal{F}_{k}\left(X_{0}, Y_{0}\right)=0, \quad k=1,2
$$

satisfying

$$
\operatorname{det}\left(\left.\frac{\partial\left(\mathcal{F}_{1}, \mathcal{F}_{2}\right)}{\partial\left(X_{0}, Y_{0}\right)}\right|_{\left(X_{0}, Y_{0}\right)=\left(X_{0}{ }^{*}, Y_{0}{ }^{*}\right)}\right) \neq 0
$$

the differential equation (1) has a periodic solution $\varepsilon u(t, \varepsilon)$ such that $u(t, \varepsilon)$ tends to the solution $u_{0}(t)$ given by

$$
u_{0}(t)=\frac{\left(-\sqrt{b_{0}} X_{0}^{*}+a_{0} Y_{0}^{*}\right) \cos \left(\sqrt{b_{0}} t\right)+\left(a_{0} X_{0}^{*}+\sqrt{b_{0}} Y_{0}^{*}\right) \sin \left(\sqrt{b_{0}} t\right)}{\sqrt{b_{0}}\left(a_{0}^{2}+b_{0}\right)}
$$

of $u^{\prime \prime \prime}+a_{0} u^{\prime \prime}+b_{0} u^{\prime}+a_{0} b_{0} u=0$ when $\varepsilon \rightarrow 0$. Note that this solution is periodic of $\operatorname{period} \frac{2 \pi}{\sqrt{b_{0}}}$.

Theorem 1 is proved in section 3. Its proof is based in the averaging theory for computing periodic orbits, see section 2. For others applications of the averaging theory to the study of limit cycles, see [3] and [11].

An application of Theorem 1 is the following.

Corollary 2. Consider the differential equation (1) where the function $F$ satisfies

$$
F\left(t, \varepsilon u, \varepsilon u^{\prime}, \varepsilon u^{\prime \prime}, \varepsilon\right)=\left(u^{2}-1\right) \sin \left(\sqrt{b_{0}} t\right)+O(\varepsilon) \text {. }
$$


Then this differential equation has four periodic solutions $\varepsilon u_{k}(t, \varepsilon)$ for $k=1,2,3,4$ tending to the periodic solutions $\varepsilon u_{k}(t)$ where

$$
\begin{array}{ll}
u_{1}(t)=-\frac{2}{\sqrt{3}} \sin \left(\sqrt{b_{0}} t\right), & u_{2}(t)=\frac{2}{\sqrt{3}} \sin \left(\sqrt{b_{0}} t\right), \\
u_{3}(t)=2 \cos \left(\sqrt{b_{0}} t\right), & u_{4}(t)=-2 \cos \left(\sqrt{b_{0}} t\right),
\end{array}
$$

of $u^{\prime \prime \prime}+a_{0} u^{\prime \prime}+b_{0} u^{\prime}+a_{0} b_{0} u=0$ when $\varepsilon \longrightarrow 0$.

Corollary 2 is proved in section 4 .

\section{BASIC RESUlts on AVERAGing theory}

In this section we present the basic results from the averaging theory that we shall need for proving the main results of this paper.

We consider the problem of the bifurcation of $T$-periodic solutions from differential systems of the form

$$
\dot{\mathbf{x}}=F_{0}(t, \mathbf{x})+\varepsilon F_{1}(t, \mathbf{x})+\varepsilon^{2} F_{2}(t, \mathbf{x}, \varepsilon),
$$

with $\varepsilon=0$ to $\varepsilon \neq 0$ sufficiently small. Here the functions $F_{0}, F_{1}: \mathbb{R} \times \Omega \rightarrow \mathbb{R}^{n}$ and $F_{2}: \mathbb{R} \times \Omega \times\left(-\varepsilon_{0}, \varepsilon_{0}\right) \rightarrow \mathbb{R}^{n}$ are $\mathcal{C}^{2}$ functions, $T$-periodic in the first variable, and $\Omega$ is an open subset of $\mathbb{R}^{n}$. The main assumption is that the unperturbed system

$$
\dot{\mathbf{x}}=F_{0}(t, \mathbf{x}),
$$

has a submanifold of periodic solutions. A solution of this problem is given using the averaging theory.

Let $\mathbf{x}(t, \mathbf{z}, \varepsilon)$ be the solution of the system (5) such that $\mathbf{x}(0, \mathbf{z}, \varepsilon)=\mathbf{z}$. We write the linearization of the unperturbed system along a periodic solution $\mathbf{x}(t, \mathbf{z}, 0)$ as

$$
\dot{\mathbf{y}}=D_{\mathbf{x}} F_{0}(t, \mathbf{x}(t, \mathbf{z}, 0)) \mathbf{y} .
$$

In what follows we denote by $M_{\mathbf{z}}(t)$ some fundamental matrix of the linear differential system (6), and by $\xi: \mathbb{R}^{k} \times \mathbb{R}^{n-k} \rightarrow \mathbb{R}^{k}$ the projection of $\mathbb{R}^{n}$ onto its first $k$ coordinates; i.e. $\xi\left(x_{1}, \ldots, x_{n}\right)=\left(x_{1}, \ldots, x_{k}\right)$.

We assume that there exists a $k$-dimensional submanifold $\mathcal{Z}$ of $\Omega$ filled with $T$-periodic solutions of (5). Then an answer to the problem of bifurcation of $T$ periodic solutions from the periodic solutions contained in $\mathcal{Z}$ for system (4) is given in the following result.

Theorem 3. Let $W$ be an open and bounded subset of $\mathbb{R}^{k}$, and let $\beta: \mathrm{Cl}(W) \rightarrow$ $\mathbb{R}^{n-k}$ be a $\mathcal{C}^{2}$ function. We assume that

(i) $\mathcal{Z}=\left\{\mathbf{z}_{\alpha}=(\alpha, \beta(\alpha)), \alpha \in \mathrm{Cl}(W)\right\} \subset \Omega$ and that for each $\mathbf{z}_{\alpha} \in \mathcal{Z}$ the solution $\mathbf{x}\left(t, \mathbf{z}_{\alpha}\right)$ of (5) is T-periodic;

(ii) for each $\mathbf{z}_{\alpha} \in \mathcal{Z}$ there is a fundamental matrix $M_{\mathbf{z}_{\alpha}}(t)$ of (6) such that the matrix $M_{\mathbf{z}_{\alpha}}^{-1}(0)-M_{\mathbf{z}_{\alpha}}^{-1}(T)$ has in the upper right corner the $k \times(n-k)$ zero matrix, and in the lower right corner $a(n-k) \times(n-k)$ matrix $\Delta_{\alpha}$ with $\operatorname{det}\left(\Delta_{\alpha}\right) \neq 0$.

We consider the function $\mathcal{F}: \mathrm{Cl}(W) \rightarrow \mathbb{R}^{k}$

$$
\mathcal{F}(\alpha)=\xi\left(\frac{1}{T} \int_{0}^{T} M_{\mathbf{z}_{\alpha}}^{-1}(t) F_{1}\left(t, \mathbf{x}\left(t, \mathbf{z}_{\alpha}\right)\right) d t\right) .
$$

If there exists $a \in W$ with $\mathcal{F}(a)=0$ and $\operatorname{det}((d \mathcal{F} / d \alpha)(a)) \neq 0$, then there is a $T$-periodic solution $\varphi(t, \varepsilon)$ of system (4) such that $\varphi(0, \varepsilon) \rightarrow \mathbf{z}_{a}$ as $\varepsilon \rightarrow 0$. 
Theorem 3 goes back to Malkin [13] and Roseau [15], for a shorter proof see [2].

\section{Proof of TheOREM 1}

Introducing the variables $(x, y, z)=\left(u, u^{\prime}, u^{\prime \prime}\right)$ we write the third-order differential equation (1) as a first-order differential system defined in an open subset $\Omega$ of $\mathbb{R}^{3}$. Thus we have the differential system

$$
\begin{aligned}
& x^{\prime}=y \\
& y^{\prime}=z \\
& z^{\prime}=-\left(a_{0} b_{0} x+b_{0} y+a_{0} z+c_{2} x^{2}+b_{1} x y+a_{1} x z\right)+\varepsilon^{2} F(t, x, y, z, \varepsilon) .
\end{aligned}
$$

Of course as before the dot denotes derivative with respect to the independent variable $t$. Doing the rescaling $(x, y, z) \rightarrow(\varepsilon X, \varepsilon Y, \varepsilon Z)$ we obtain the system

$$
\begin{aligned}
& x^{\prime}=y \\
& y^{\prime}=z \\
& z^{\prime}=-a_{0} b_{0} x-b_{0} y-a_{0} z+\varepsilon\left(-c_{2} x^{2}-b_{1} x y-a_{1} x z+F_{0}(t, x, y, z)\right)+O\left(\varepsilon^{2}\right) .
\end{aligned}
$$

Recall that $F_{0}(t, x, y, z)$ has been defined in the statement of Theorem 1 . Note that we have denoted the new variables $(\mathrm{X}, \mathrm{Y}, \mathrm{Z})$ again by $(\mathrm{x}, \mathrm{y}, \mathrm{z})$. System $(9)$ with $\varepsilon=0$ will be called the unperturbed system, otherwise we have the perturbed system. The unperturbed system has a unique singular point, the origin. Since $b_{0}>0$, the origin has one pair of imaginary eigenvalues and one real eigenvalue, more precisely the eigenvalues are $-a_{0}, \sqrt{b_{0}} i,-\sqrt{b_{0}} i$. We shall write system (9) in such a way that the linear part at the origin will be in its real Jordan normal form. Then, doing the change of variables $(x, y, z) \rightarrow(X, Y, Z)$ given by

$$
\left(\begin{array}{l}
X \\
Y \\
Z
\end{array}\right)=\left(\begin{array}{ccc}
0 & a_{0} & 1 \\
a_{0} \sqrt{b_{0}} & \sqrt{b_{0}} & 0 \\
b_{0} & 0 & 1
\end{array}\right)\left(\begin{array}{l}
x \\
y \\
z
\end{array}\right)
$$

the differential system (9) becomes

$$
\begin{aligned}
& X^{\prime}=-\sqrt{b_{0}} Y+\varepsilon G_{1}(t, X, Y, Z)+O\left(\varepsilon^{2}\right), \\
& Y^{\prime}=\sqrt{b_{0}} X, \\
& Z^{\prime}==-a_{0} Z+\varepsilon G_{3}(t, X, Y, Z)+O\left(\varepsilon^{2}\right),
\end{aligned}
$$


with

$$
\begin{aligned}
G_{1}(t, X, Y, Z)= & \frac{\left(a_{1} b_{0}+a_{0} b_{1}-c_{2}\right) X^{2}}{\left(a_{0}^{2}+b_{0}\right)^{2}}+\frac{a_{0}\left(a_{0} a_{1} b_{0}-b_{0} b_{1}-a_{0} c_{2}\right) Y^{2}}{b_{0}\left(a_{0}^{2}+b_{0}\right)^{2}}- \\
& \frac{\left(a_{0}^{2} a_{1}-a_{0} b_{1}+c_{2}\right) Z^{2}}{\left(a_{0}^{2}+b_{0}\right)^{2}}+\frac{\left(-a_{0}^{2} b_{1}+b_{0} b_{1}+2 a_{0}\left(-a_{1} b_{0}+c_{2}\right)\right) X Y}{\sqrt{b_{0}}\left(a_{0}^{2}+b_{0}\right)^{2}}+ \\
& \left.\frac{\left(a_{0}^{2} a_{1}-a_{1} b_{0}-2 a_{0} b_{1} 2 c_{2}\right) X Z}{\left(a_{0}^{2}+b_{0}\right)^{2}}+\frac{\left(-\left(a_{0}^{2}-b_{0}\right)\left(a_{0} a_{1}-b_{1}\right)-2 a_{0} c_{2}\right) Y Z}{\sqrt{b_{0}}\left(a_{0}^{2}+b_{0}\right)}\right)+ \\
& F_{0}(t, a(t), b(t), c(t)) \\
G_{3}(t, X, Y, Z)= & \frac{\left(a_{1} b_{0}+a_{0} b_{1}-c_{2}\right) X^{2}}{\left(a_{0}^{2}+b_{0}\right)^{2}}+\frac{a_{0}\left(a_{0} a_{1} b_{0}-b_{0} b_{1}-a_{0} c_{2}\right) Y^{2}}{b_{0}\left(a_{0}^{2}+b_{0}\right)^{2}}- \\
& \frac{\left(a_{0}^{2} a_{1}-a_{0} b_{1}+c_{2}\right) Z^{2}}{\left(a_{0}^{2}+b_{0}\right)^{2}}+\frac{\left(-a_{0}^{2} b_{1}+b_{0} b_{1}+2 a_{0}\left(-a_{1} b_{0}+c_{2}\right)\right) X Y}{\sqrt{b_{0}}\left(a_{0}^{2}+b_{0}\right)^{2}}+ \\
& \left.\frac{\left(a_{0}^{2} a_{1}-a_{1} b_{0}-2 a_{0} b_{1}+2 c_{2}\right) X Z}{\left(a_{0}^{2}+b_{0}\right)^{2}}+\frac{\left(-\left(a_{0}^{2}-b_{0}\right)\left(a_{0} a_{1}-b_{1}\right)-2 a_{0} c_{2}\right) Y Z}{\sqrt{b_{0}}\left(a_{0}^{2}+b_{0}\right)}\right)+ \\
& F_{0}(t, a(t), b(t), c(t))
\end{aligned}
$$

and

$$
\begin{aligned}
& a(t)=-\frac{\sqrt{b_{0}} X-a_{0} Y-\sqrt{b_{0}} Z}{\sqrt{b_{0}}\left(a_{0}^{2}+b_{0}\right)}, \\
& b(t)=\frac{a_{0} X+\sqrt{b_{0}} Y-a_{0} Z}{a_{0}^{2}+b_{0}}, \\
& c(t)=\frac{b_{0} X-a_{0} \sqrt{b_{0}} Y+a_{0}^{2} Z}{a_{0}^{2}+b_{0}} .
\end{aligned}
$$

Note that the linear part of the differential system (10) at the origin is in its real normal form of Jordan. We shall apply Theorem 3 to the differential system (10). We note that system (10) can be written as system (4) taking

$$
\mathbf{x}=\left(\begin{array}{c}
X \\
Y \\
Z
\end{array}\right), \quad t=t, \quad F_{0}(t, \mathbf{x})=\left(\begin{array}{c}
-\sqrt{b_{0}} Y \\
\sqrt{b_{0}} X \\
-a_{0} Z
\end{array}\right)
$$

and

$$
F_{1}(t, \mathbf{x})=\left(\begin{array}{c}
G_{1}(t, X, Y, Z) \\
0 \\
G_{3}(t, X, Y, Z)
\end{array}\right) .
$$

We shall study the periodic solutions of system (5) in our case, i.e. the periodic solutions of system (10) with $\varepsilon=0$. These periodic solutions are

$$
\left(\begin{array}{c}
X(t) \\
Y(t) \\
Z(t)
\end{array}\right)=\left(\begin{array}{c}
X_{0} \cos \left(\sqrt{b_{0}} t\right)-Y_{0} \sin \left(\sqrt{b_{0}} t\right) \\
Y_{0} \cos \left(\sqrt{b_{0}} t\right)+X_{0} \sin \left(\sqrt{b_{0}} t\right) \\
0
\end{array}\right) .
$$

This set of periodic solutions has dimension two, all having the same period $\frac{2 \pi}{\sqrt{b_{0}}}$.

To look for the periodic solutions of our equation (10) we must calculate the zeros $\mathbf{z}=\left(X_{0}, Y_{0}\right)$ of the system $\mathcal{F}(\mathbf{z})=0$, where $\mathcal{F}(\mathbf{z})$ is given by (7). The fundamental 
matrix $M(t)$ of the differential system (10) with $\varepsilon=0$ along any periodic solution is

$$
M(t)=M_{\mathbf{z}}(t)=\left(\begin{array}{ccc}
\cos \left(\sqrt{b_{0}} t\right) & -\sin \left(\sqrt{b_{0}} t\right) & 0 \\
\sin \left(\sqrt{b_{0}} t\right) & \cos \left(\sqrt{b_{0}} t\right) & 0 \\
0 & 0 & \exp \left(-a_{0} t\right)
\end{array}\right) .
$$

The inverse matrix of $M(t)$ is

$$
M^{-1}(t)=\left(\begin{array}{ccc}
\cos \left(\sqrt{b_{0}} t\right) & \sin \left(\sqrt{b_{0}} t\right) & 0 \\
-\sin \left(\sqrt{b_{0}} t\right) & \cos \left(\sqrt{b_{0}} t\right) & 0 \\
0 & 0 & \exp \left(a_{0} t\right)
\end{array}\right) .
$$

It verifies

$$
M^{-1}(0)-M^{-1}\left(\frac{2 \pi}{\sqrt{b_{0}}}\right)=\left(\begin{array}{ccc}
0 & 0 & 0 \\
0 & 0 & 0 \\
0 & 0 & 1-\exp \left(\frac{2 a_{0} \pi}{\sqrt{b_{0}}}\right)
\end{array}\right) .
$$

Consequently all the assumptions of Theorem 3 are satisfied. Therefore we must study the zeros of the system $\mathcal{F}(\mathbf{z})=0$ of two equations with two unknowns, where $\mathcal{F}$ is given in the statement of Theorem 3. More precisely, we have $\mathcal{F}(\mathbf{z})=$ $\left(\mathcal{F}_{1}\left(X_{0}, Y_{0}\right), \mathcal{F}_{2}\left(X_{0}, Y_{0}\right)\right)$ where

$$
\begin{aligned}
& \mathcal{F}_{1}\left(X_{0}, Y_{0}\right)=\frac{\sqrt{b_{0}}}{2 \pi} \int_{0}^{\frac{2 \pi}{\sqrt{b_{0}}}} \cos \left(\sqrt{b_{0}} t\right)(F(t, A(t), B(t), C(t))+D(t)) d t, \\
& \mathcal{F}_{2}\left(X_{0}, Y_{0}\right)=-\frac{\sqrt{b_{0}}}{2 \pi} \int_{0}^{\frac{2 \pi}{\sqrt{b_{0}}}} \sin \left(\sqrt{b_{0}} t\right)(F(t, A(t), B(t), C(t))+D(t)) d t,
\end{aligned}
$$

with $A(t), B(t), C(t)$ and $D(t)$ as in the statement of Theorem 1.

The zeros $\left(X_{0}^{*}, Y_{0}^{*}\right)$ of system

$$
\left(\begin{array}{c}
\mathcal{F}_{1}\left(X_{0}, Y_{0}\right) \\
\mathcal{F}_{2}\left(X_{0}, Y_{0}\right)
\end{array}\right)=\left(\begin{array}{l}
0 \\
0
\end{array}\right)
$$

with respect to the variables $X_{0}$ and $Y_{0}$ provide periodic solutions of system (10) with $\varepsilon \neq 0$ sufficiently small if they are simple, i.e. if

$$
\operatorname{det}\left(\left.\frac{\partial\left(\mathcal{F}_{1}, \mathcal{F}_{2}\right)}{\partial\left(X_{0}, Y_{0}\right)}\right|_{\left(X_{0}, Y_{0}\right)=\left(X_{0}^{*}, Y_{0}^{*}\right)}\right) \neq 0 .
$$

Going back through the change of variable, for every simple zero $\left(X_{0}^{*}, Y_{0}^{*}\right)$ of system (11), we obtain a $\frac{2 \pi}{\sqrt{b_{0}}}$ periodic solution $\varepsilon u(t, \varepsilon)$ of the differential equation (1) for $\varepsilon \neq 0$ sufficiently small such that $u(t, \varepsilon)$ tends to the periodic solution

$$
u_{0}(t)=\frac{\left(-\sqrt{b_{0}} X_{0}^{*}+a_{0} Y_{0}^{*}\right) \cos \left(\sqrt{b_{0}} t\right)+\left(a_{0} X_{0}^{*}+\sqrt{b_{0}} Y_{0}^{*}\right) \sin \left(\sqrt{b_{0}} t\right)}{\sqrt{b_{0}}\left(a_{0}^{2}+b_{0}\right)}
$$


of $u^{\prime \prime \prime}+a_{0} u^{\prime \prime}+b_{0} u^{\prime}+a_{0} b_{0} u=0$ when $\varepsilon \rightarrow 0$. Note that this solution is periodic of period $\frac{2 \pi}{\sqrt{b_{0}}}$. This completes the proof of Theorem 1 .

\section{Proof of Corollary 2}

We must apply Theorem 1 with $F_{0}(t, x, y, z, v)=\left(x^{2}-1\right) \sin \left(\sqrt{b_{0}} t\right)$. After a tedious but easy computations the functions $\mathcal{F}_{1}$ and $\mathcal{F}_{2}$ of Theorem 1 are

$$
\begin{aligned}
& \mathcal{F}_{1}\left(X_{0}, Y_{0}\right)=\frac{\left(-\sqrt{b_{0}} X_{0}+a_{0} Y_{0}\right)\left(a_{0} X_{0}+\sqrt{b_{0}} Y_{0}\right)}{4 b_{0}\left(a_{0}^{2}+b_{0}\right)^{2}}, \\
& \mathcal{F}_{2}\left(X_{0}, Y_{0}\right)=-\frac{-a_{0}^{4} b_{0}+4 a_{0} \sqrt{b_{0}} X_{0} Y_{0}+a_{0}^{2}\left(-8 b_{0}^{2}+3 X_{0}^{2}+Y_{0}^{2}\right)+b_{0}\left(-4 b_{0}^{2}+X_{0}^{2}+3 Y_{0}^{2}\right)}{8 b_{0}\left(a_{0}^{2}+b_{0}\right)^{2}} .
\end{aligned}
$$

System $\mathcal{F}_{1}=\mathcal{F}_{2}=0$ has four solutions $\left(X_{0}^{*}, Y_{0}^{*}\right)$ given by

$$
\left(-\frac{2 a_{0} \sqrt{b_{0}}}{\sqrt{3}},-\frac{2 b_{0}}{\sqrt{3}}\right),\left(\frac{2 a_{0} \sqrt{b_{0}}}{\sqrt{3}}, \frac{2 b_{0}}{\sqrt{3}}\right),\left(-2 b_{0}, 2 a_{0} \sqrt{b_{0}}\right),\left(2 b_{0},-2 a_{0} \sqrt{b_{0}}\right) .
$$

Since the Jacobian

$$
\operatorname{det}\left(\left.\frac{\partial\left(\mathcal{F}_{1}, \mathcal{F}_{2}\right)}{\partial\left(X_{0}, Y_{0}\right)}\right|_{\left(X_{0}, Y_{0}\right)=\left(X_{0}^{*}, Y_{0}^{*}\right)}\right)
$$

for these four solutions $\left(X_{0}^{*}, Y_{0}^{*}\right)$ is $\frac{1}{4 b_{0}\left(a_{0}^{2}+b_{0}\right)}, \frac{1}{4 b_{0}\left(a_{0}^{2}+b_{0}\right)},-\frac{1}{4 b_{0}\left(a_{0}^{2}+b_{0}\right)}$, $-\frac{1}{4 b_{0}\left(a_{0}^{2}+b_{0}\right)}$ respectively, we obtain using Theorem 1 the four solutions given in the statement of the corollary.

\section{ACKNOWLEDGEMENTS}

The first author is partially supported by a MICIIN/FEDER grant MTM2008 03437, by a CIRIT grant number 2009SGR-410 and by ICREA Academia.

\section{REFERENCES}

[1] R. BAmON, Quadratic vector fields in the plane have a finite number of limit cycles, Inst. Hautes Etudes Sci. Publ. Mat. 64 (1986), 111-142.

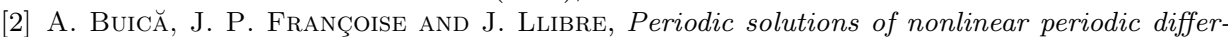
ential systems with a small parameter, Comm. on Pure and Appl. Anal. 6 (2007), 103-111.

[3] C. A. Buzzi, J. Llibre, J. C. Medrado and J. Torregrosa, Bifurcation of limit cycles from a center in $\mathbb{R}^{4}$ in resonance 1: N, Dyn. Sys. : An Int. Journal 24 (2009), 123-137.

[4] G. Chen And T. Ueta, Yet another chaotic attractor, Int. J. Bifur. Chaos 9 (1999), 14651466.

[5] F.S. Dias AND L.F. Mello, Nonlinear analysis of a quadratic system obtained from a scalar third order differential equation, Electron. J. Diff. Equ. 161 (2010), 1-25.

[6] J. ECALLE, Introduction aux fonctions analysables et preuve constructive de la conjecture de Dulac (French), Hermann, Paris, 1992.

[7] A. Ferragut, J. Llibre and C. Pantazi, Polynomial vector fields in $\mathbb{R}^{3}$ with infinitely many limit cycles, submitted, 2010.

[8] Y. Ilyashenko, Finiteness Theorems for limit cycles, American Mathematical Society, Providence, RI, 1993.

[9] J. Lu And G. Chen, A new chaotic attractor coined, Int. J. Chaos 12 (2002), 659-661.

[10] C. LiU, T. LiU, L. Liu And K. LiU, A new chaotic, Chaos, Solitons and Fractals 22 (2004), 1031-1038.

[11] J. Llibre, A. C. Mereu And M. A. Teixeira, Limit cycles of resonant four-dimensional polynomial system, Dyn. Sys. : An Int. Journal 25 (2010), 145-158. 
[12] E.N. Lorenz, Deterministic nonperiodic flow, J. Atmos. Sci. 20 (1963), 130-141.

[13] I.G. Malkin, Some problems of the theory of nonlinear oscillations, (Russian) Gosudarstv. Izdat. Tehn.-Teor. Lit., Moscow, 1956.

[14] T. Rikitake, Oscillations of a system of disk dynamos, Proc. R. Cambridge Philos. Soc. 54 (1958), 89-105

[15] M. Roseau, Vibrations non linéaires et théorie de la stabilité, (French) Springer Tracts in Natural Philosophy, Vol. 8, Springer-Verlag, Berlin-New York, 1966.

[16] E. RössLer, An equation for continuous chaos, Phys. Lett. A, 57 (1976), 397-398.

1 Departament de Matematiques, Universitat Autònoma de Barcelona, 08193 Bellaterra, Barcelona, Catalonia, Spain

E-mail address: jllibre@mat.uab.cat

2 Department of Mathematics, University of Annaba, Elhadjar 23 Annaba, Algeria

E-mail address: makhloufamar@yahoo.fr 